\title{
Bayesian analysis of differential effects in multi-group regression methods
}

\author{
Adrian Quintero', Geert Verbeke ${ }^{1}$, Luk Bruyneel ${ }^{2}$ and Emmanuel Lesaffre ${ }^{1}$ \\ ${ }^{1}$ I-BioStat, KU Leuven, Leuven, Belgium. \\ ${ }^{2}$ Department of Public Health and Primary Care, KU Leuven, Leuven, Belgium.
}

\begin{abstract}
In regression analysis, the data sample is often composed of diverse sub-populations such as ethnicities and geographical regions. In multiple application areas, it is important to identify the groups where each covariate has a positive, negative or null impact on the response. If the number of sub-populations is small, a full interaction model may be fit with group-specific covariate effects. However, if the number of groups is very large, for example, hospitals or other clustering units, such a model is not identifiable. Therefore, we propose a prior distribution which combines the information across sub-populations with a similar covariate effect. This Bayesian analysis of differential effects (BADE) classifies the group-specific covariate effects as positive, negative or null. Besides allowing the analysis of differential effects for many sub-populations, the proposed approach improves substantially the identification of important interactions in cases with few groups. This is illustrated via simulations. The procedure is motivated on, and applied to, a large study related to patients' satisfaction with hospitals, where we show that classifying group-specific covariate effects based on methods such as mixed-effects models may be strongly misleading.
\end{abstract}

Key words: differential effects, full interaction model, latent variable, MCMC methods, multiple groups

Received May 2019; revised August 2019; accepted September 2019

\section{Introduction}

In regression analysis, the response is usually modelled as a function of the explanatory variables assuming that the effect of each covariate is the same for all observations. Such a model can be expressed as

$$
y_{i}=\beta_{0} x_{0, i}+\beta_{1} x_{1, i}+\cdots+\beta_{p} x_{p, i}+\varepsilon_{i},
$$

where the covariate $x_{0}$ corresponds to the intercept and the error is assumed to follow $\varepsilon_{i} \sim \mathcal{N}\left(0, \sigma^{2}\right)$ for $i=1, \ldots, n$. However, data often come from populations with well-defined sub-groups such as geographical regions, distinct levels of education, hospitals or other clustering units.

Address for correspondence: Adrian Quintero, I-BioStat, KU Leuven, Kapucijnenvoer 35, B3000 Leuven, Belgium.

E-mail: aquintero@icfes.gov.co

(C) 2019 SAGE Publications

$10.1177 / 1471082 X 19881844$ 


\section{Adrian Quintero et al.}

In multiple application areas, it is important to identify the sub-populations where each covariate has a positive, negative or null effect. Such is the case of clinical trials where the investigators are often interested in determining whether the effectiveness of a treatment is valid in different sub-groups of patients (Dixon and Simon, 1991; Schnell et al., 2016); the analysis of differential molecular profiles in cancer when the samples come from multiple sub-populations (Ishwaran and Rao, 2005); and psychometric tests where it is important to verify that each item is measuring the same abilities across geographical regions, ethnicities, etc. (Zumbo, 2007). In this article, we use the terms sub-populations and sub-groups interchangeably.

In the motivating RN4CAST study (Sermeus et al., 2011), the patients rated their hospitals as a measure of overall satisfaction with care. Additionally, the individuals filled in 16 survey items related to various experiences during their stay. Thus, it is of interest to determine which of the 16 items have a positive or negative effect in each hospital in order to define hospital-specific policies to improve overall patient satisfaction.

If the number of sub-populations in the study is small, a full interaction model can be considered of the form

$$
y_{i(g)}=\beta_{0(g)} x_{0, i(g)}+\beta_{1(g)} x_{1, i(g)}+\cdots+\beta_{p(g)} x_{p, i(g)}+\varepsilon_{i(g)},
$$

where $y_{i(g)}$ is the response for individual $i=1, \ldots, n_{g}$ in sub-group $g=1, \ldots, G$ and $\varepsilon_{i(g)} \sim \mathcal{N}\left(0, \sigma^{2}\right)$. The regressor $x_{j, i(g)}$ corresponds to the interaction between covariate $x_{j, i}$ and group membership. Hence, the effect of each covariate $x_{j}(j=$ $0, \ldots, p)$ in sub-group $g$ can be judged based on $\beta_{j(g)}$. Note that some predictors in model (1.2) may be included without differential effects, but in the notation we assume that interactions are incorporated for all covariates to ease the presentation of the method.

Bayesian sub-group analysis considers model (1.2) in the context of clinical trials, but including differential effects only for the treatment indicator covariate (Pocock et al., 2002; Liu et al., 2017). Based on such a model, the effectiveness of the treatment can be verified for different clinically important sub-groups of the population. Similarly, economic models that encompass macro- and micro-regression estimates for distinct industries and financial sectors (Pakes, 1983) are based on model (1.2).

However, the full interaction model (1.2) is not identifiable when the number of sub-populations is very large (e.g., hospitals or other clustering units). For those cases, a random-effects model could be considered, allowing for random group-specific coefficients in all covariates. Nonetheless, this alternative poses multiple difficulties: (a) the normality assumption for the random-effects may not hold in practice and lead to biased estimates for the random-effects themselves (Verbeke and Lesaffre, 1996); (b) estimating the covariance matrix of the $p$-dimensional random-effects vector becomes problematic when the number of covariates with differential effects is large (Verbeke and Molenberghs, 2000, Chapter 5.6.1) and (c) in mixed models, the random-effects are pooled altogether towards the overall mean 
(Carlin and Louis, 1996), causing important effects to appear as non-important or vice versa. This last issue was pointed out by Sargent and Hodges (1997) when fitting many interaction terms via hierarchical models, in which case shrinking the coefficients towards different centres should be considered.

Therefore, we propose a Bayesian approach which combines information only among sub-groups with a similar covariate effect. This method enables the identification of sub-populations with a positive, negative and null impact, allowing for this inter-group variability to differ across predictors. As illustrated via simulations, our method is also advantageous in situations with few sub-populations compared to model (1.2), because the proposed approach combines the information across sub-groups.

Using a Bayesian hierarchical model, we assume that for a given covariate $x_{j}$, the interaction coefficients across sub-groups $\beta_{j(1)}, \ldots, \beta_{j(G)}$ in model (1.2) can be classified into three components: sub-populations where the effect of the covariate is negative, sub-groups where there is no effect on the response and sub-populations where the impact is positive. Thence, two sub-groups are said to have a similar covariate effect if they belong to the same component, that is, if the regressor has a negative/null/positive effect in both sub-groups. On the other hand, we refer to differential effects of a covariate when the sub-populations are classified in more than one component.

By introducing latent variables (Tanner and Wong, 1987), we classify the sub-populations into the three defined components and combine the information from sub-groups with a similar covariate effect. Thus, based on posterior inference, we establish the classification of the interactions which is most supported by the data, in order to study possible differential effects of the covariates.

The rest of the article is organized as follows. In Section 2, we describe the proposed Bayesian method to analyse differential effects in multi-group studies. The Gibbs sampling method for posterior inference is discussed in Section 3. A simulation study is carried out in Section 4 to illustrate the advantages of the proposed methodology and to show that using a random-effects model to classify group-specific covariate effects may be strongly misleading. In Section 5, we analyse which patient experiences have an impact on overall satisfaction with hospitals in the RN4CAST study. The article concludes with a general discussion in Section 6.

\section{Study of differential effects}

We implement an approach for Bayesian analysis of differential effects (BADE) that begins with the full interaction model (1.2) and combines the information across sub-populations with a similar covariate effect. Notice that in model (1.2) we are not using effect coding nor reference coding, but rather a model without a baseline. This is to avoid choosing a reference group and a relative interpretation of the coefficients given the selected baseline. As discussed in Section 6, the approach can be adapted to the case where there is a predefined reference group. 


\section{Adrian Quintero et al.}

\subsection{The method}

For each explanatory variable $x_{j}$, we introduce latent variables $\gamma_{j(g)} \in\{-1,0,+1\}$ $(g=1, \ldots, G)$ to classify the interaction coefficients $\beta_{j(g)}$ in the three defined components: the covariate $x_{j}$ has no impact in sub-group $g$ when $\gamma_{j(g)}=0$, the effect is negative if $\gamma_{j(g)}=-1$ and the covariate effect is positive when $\gamma_{j(g)}=+1$. The BADE approach begins with model (1.2) but assumes that the interaction coefficients for each covariate $x_{j}$ follow a mixture distribution with three components: a normal density truncated above zero, a spike at zero and a normal density truncated below zero. More specifically,

$$
p\left(\beta_{j(g)} \mid \gamma_{j(g)}\right)= \begin{cases}N^{-}\left(\mu_{j(-)}, \sigma_{j(-)}^{2}\right) & \text { if } \gamma_{j(g)}=-1 \\ \delta_{0}\left(\beta_{j(g)}\right) & \text { if } \gamma_{j(g)}=0 \\ N^{+}\left(\mu_{j(+)}, \sigma_{j(+)}^{2}\right) & \text { if } \gamma_{j(g)}=+1\end{cases}
$$

where $\mu_{j(-)}<0, \mu_{j(+)}>0(j=0, \ldots, p)$ and $\delta_{0}($.$) is the Dirac delta function at 0$. The graphical representation of this prior is depicted in Figure 1 for a specific case taking $\mu_{j(-)}=-0.6, \sigma_{j(-)}=0.2, \mu_{j(+)}=0.3, \sigma_{j(+)}=0.1$ and $p\left(\gamma_{j(g)}=-1\right)=p\left(\gamma_{j(g)}=\right.$ $0)=p\left(\gamma_{j(g)}=+1\right)=1 / 3$.

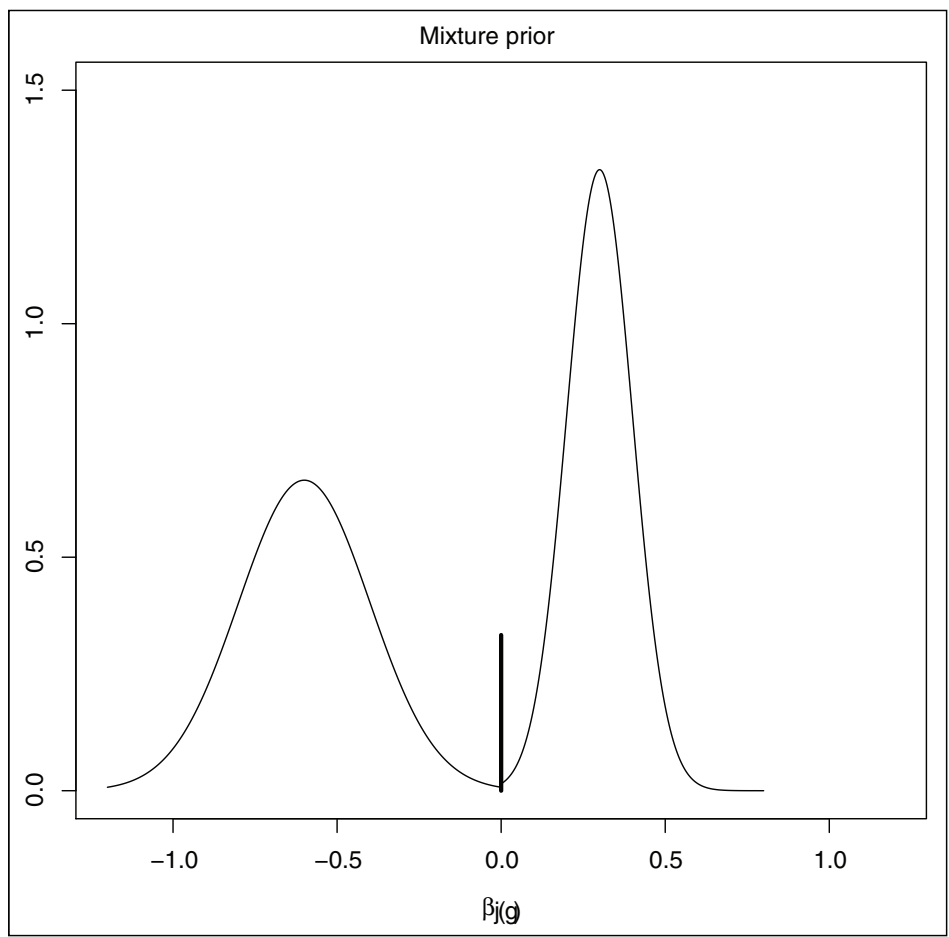

Figure 1 Proposed mixture prior (2.1) with three components for BADE approach 
The proposed prior (2.1) allows us to study differential effects distinguishing sub-groups where each covariate has a negative/null/positive impact based on the latent variables $\gamma_{j(g)}$. Additionally, the information for the sub-groups with a similar covariate effect is combined through the truncated normal components in the prior, where the estimated effects are pooled towards the mean of each component.

The information regarding the classification of all interactions in the BADE method is contained in the matrix $\boldsymbol{\gamma}=\left(\boldsymbol{\gamma}_{0}, \ldots, \boldsymbol{\gamma}_{p}\right)$ where $\boldsymbol{\gamma}_{j}^{\prime}=\left(\gamma_{j(1)}, \ldots, \gamma_{j(G)}\right)$ for $j=0, \ldots, p$. Following up the definition of the parameters, the proportion of sub-populations where each covariate $x_{j}$ has no impact is $\pi_{j(0)}=\operatorname{Pr}\left(\gamma_{j(g)}=0\right)$, whereas the overall proportions with a negative and positive effect are respectively $\pi_{j(-)}=\operatorname{Pr}\left(\gamma_{j(g)}=-1\right)$ and $\pi_{j(+)}=\operatorname{Pr}\left(\gamma_{j(g)}=+1\right)$. The model specification is completed by assuming independence for $\gamma_{j(g)}$ 's across covariates and sub-populations conditionally on $\pi=\left(\boldsymbol{\pi}_{1}, \ldots, \boldsymbol{\pi}_{p}\right)$ where $\boldsymbol{\pi}_{j}^{\prime}=\left(\pi_{j(-)}, \pi_{j(0)}, \pi_{j(+)}\right)$.

As discussed in Section 1, in mixed models the random-effects are pooled altogether towards the overall mean, causing important effects to appear as non-important or vice versa. In contrast, the proposed prior (2.1) avoids this by shrinking the coefficients towards different centres depending on the effect of the covariate in each sub-group.

Parmigiani et al. (2002) proposed a three-component mixture for molecular classification in cancer. The authors considered a normal distribution in the mixture and two uniform densities in the lower and upper tails, respectively. Fixing the mean of the normal component to zero would allow us to classify the interactions similarly as in (2.1). However, the normal component leads to shrinkage towards zero, causing important effects to appear as non-important, a similar phenomenon as in mixed models. In addition, the method does not borrow strength across sub-populations with a similar covariate effect because the lower and upper components follow uniform densities.

\subsection{Prior specification}

For the BADE approach, we centre the covariates and the response and rescale them in units of standard deviation. In order to obtain conditional conjugacy for the mean parameters of the truncated normal components in (2.1), a possibility would be to select truncated negative and positive normal distributions, for example, $\mu_{j(-)} \mid \sigma \sim N^{-}\left(0, r \sigma^{2}\right)$ and $\mu_{j(+)} \mid \sigma \sim N^{+}\left(0, r \sigma^{2}\right)$ respectively, with $r>0$. However, the null model $\left(\beta_{j(g)}=0\right.$ for all covariates and sub-groups) is favoured with such a prior as $r \rightarrow \infty$, which is a form of the Bartlett-Lindley paradox (Bartlett, 1957). This is discussed in more detail in the appendix. On the other hand, selecting small $r$ may shrink the mean parameters $\mu_{j(-)}$ and $\mu_{j(+)}$ towards zero. Therefore, we rather assign uniform prior densities as

$$
\mu_{j(-)}\left|\sigma \sim U\left(r_{j(-)} \sigma, 0\right), \quad \mu_{j(+)}\right| \sigma \sim U\left(0, r_{j(+)} \sigma\right),
$$


with $r_{j(-)}<0$ and $r_{j(+)}>0$ for $j=0, \ldots, p$. Notice that we allow for different parameters $r_{j(-)}$ and $r_{j(+)}$ for each covariate and for each of the two truncated normal components in the mixture. Additionally, we assign hyperpriors for these parameters as follows

$$
r_{j(-)} \sim N^{-}(0, r), \quad r_{j(+)} \sim N^{+}(0, r) .
$$

Different values of $r$ are compared in the simulation study in Section 4. We select uniform priors for $\sigma_{j(-)}$ and $\sigma_{j(+)}$, the standard deviations of the truncated normal components in the mixture (2.1). The range of these uniform priors is chosen in a scale-independent manner. For this, we take into account that when $\sigma_{j(+)}=\mu_{j(+)} / 3$, the density for the missing part in the positive truncated normal component in the prior is close to zero. The same occurs for the negative truncated normal component when $\sigma_{j(-)}=-\mu_{j(-)} / 3$. Figure 1 presents an example where $\sigma_{j(+)}=\mu_{j(+)} / 3$ and $\sigma_{j(-)}=$ $-\mu_{j(-)} / 3$. We think that it is reasonable to select $\sigma_{j(-)} \sim U\left(0,-r_{j(-)} \sigma\right)$ and $\sigma_{j(+)} \sim$ $U\left(0, r_{j(+)} \sigma\right)$ as priors, so the standard deviations can be as large as $-\mu_{j(-)}$ and $\mu_{j(+)}$ in (2.2), respectively. These ranges are quite large (compared to $\sigma_{j(-)}=-\mu_{j(-)} / 3$ and $\left.\sigma_{j(+)}=\mu_{j(+)} / 3\right)$ and allow for flexibility in the model. In the simulation study and the application to patient satisfaction, the estimated standard deviations were always far inside these boundaries.

Following standard convention, we choose $\sigma^{2} \sim \operatorname{IG}(b, c)$ for the residual variance. To complete this prior specification, the component probabilities vector is assigned a Dirichlet prior for each covariate, that is, $\pi_{j} \sim \operatorname{Dir}\left(a_{j(-)}, a_{j(0)}, a_{j(+)}\right)$. Note that the prior distribution of the latent variables $p(\boldsymbol{\gamma})=p\left(\boldsymbol{\gamma}_{0}\right) \times \cdots \times p\left(\boldsymbol{\gamma}_{p}\right)$ depends on the hyperparameters assigned to $\pi_{j}$ 's through the Dirichlet distribution. If we select $a_{j(-)}=a_{j(0)}=a_{j(+)}$ for every covariate, the same prior probability is assigned to each of the three components in (2.1).

Based on the observed $\mathbf{y}$, the posterior distribution $p(\boldsymbol{\gamma} \mid \mathbf{y}) \propto p(\mathbf{y} \mid \boldsymbol{\gamma}) p(\boldsymbol{\gamma})$ updates the prior probabilities of the possible classifications $\gamma$. The purpose of our approach is to identify the classification of the interactions that is most supported by the data whilst combining the information across sub-populations with a similar covariate effect. We now proceed to discuss the Gibbs sampling procedure to explore the posterior distribution of the parameters and latent variables in the model.

\section{Posterior inference}

After specifying model (2.1) based on the defined latent variables, we need to establish the classification that is most supported by the data for the covariates, that is, where $p(\gamma \mid \mathbf{y})$ puts most weight. This can be done by exploring the posterior distribution $p\left(\boldsymbol{\beta}, \boldsymbol{\mu}_{(-)}, \boldsymbol{\mu}_{(+)}, \boldsymbol{\sigma}_{(-)}, \boldsymbol{\sigma}_{(+)}, \mathbf{r}_{(-)}, \mathbf{r}_{(+)}, \boldsymbol{\pi}, \boldsymbol{\gamma}, \sigma \mid \mathbf{y}\right)$ via the Gibbs sampler. The matrix $\boldsymbol{\beta}=\left(\boldsymbol{\beta}_{0}, \ldots, \boldsymbol{\beta}_{p}\right)$ contains all the regression coefficients with $\boldsymbol{\beta}_{j}^{\prime}=\left(\beta_{j(1)}, \ldots, \beta_{j(G)}\right)$. 
The other vectors are defined as $\boldsymbol{\mu}_{(-)}^{\prime}=\left(\mu_{0(-)}, \ldots, \mu_{p(-)}\right), \boldsymbol{\mu}_{(+)}^{\prime}=\left(\mu_{0(+)}, \ldots, \mu_{p(+)}\right)$, $\boldsymbol{\sigma}_{(-)}^{\prime}=\left(\sigma_{0(-)}, \ldots, \sigma_{p(-)}\right), \boldsymbol{\sigma}_{(+)}^{\prime}=\left(\sigma_{0(+)}, \ldots, \sigma_{p(+)}\right), \mathbf{r}_{(-)}^{\prime}=\left(r_{0(-)}, \ldots, r_{p(-)}\right)$ and $\mathbf{r}_{(+)}^{\prime}=$ $\left(r_{0(+)}, \ldots, r_{p(+)}\right)$. The matrices $\boldsymbol{\pi}$ and $\boldsymbol{\gamma}$ are defined as in Section 2.1.

After obtaining a Gibbs sample of size $m$, the information regarding the study of differential effects is extracted from $\boldsymbol{\gamma}^{1}, \ldots, \boldsymbol{\gamma}^{m}$ which converges in distribution to $\boldsymbol{\gamma} \sim p(\boldsymbol{\gamma} \mid \mathbf{y})$. The Gibbs procedure consists on sampling from the following conditional distributions:

1. To begin, the component probabilities vector is sampled for each covariate from

$$
\boldsymbol{\pi}_{j}^{k} \sim p\left(\boldsymbol{\pi}_{j}^{k} \mid \mathbf{y}, \boldsymbol{\gamma}^{k}\right)=\operatorname{Dir}\left(I_{j(-)}^{k}+a_{j(-)}, I_{j(0)}^{k}+a_{j(0)}, I_{j(+)}^{k}+a_{j(+)}\right),
$$

where the parameters of the Dirichlet distribution relate to the number of sub-populations in each of the three components, namely, $I_{j(-)}^{k}=\sum_{g} I\left\{\gamma_{j(g)}^{k}=\right.$ $-1\}, I_{j(0)}^{k}=\sum_{g} I\left\{\gamma_{j(g)}^{k}=0\right\}$ and $I_{j(+)}^{k}=\sum_{g} I\left\{\gamma_{j(g)}^{k}=+1\right\}$. Hence, the expected value of each probability in $\boldsymbol{\pi}_{j}$ increases with the number of sub-populations that are classified in the corresponding component, for example, the expected value of $\pi_{j(-)}^{k}$ increases with $I_{j(-)}^{k}$.

Prior knowledge can be incorporated into the BADE approach in case historical or experts' information is available. For instance, as observed in (3.1), the information given by $a_{j(-)}, a_{j(0)}$ and $a_{j(+)}$ is equivalent to adding extra data to the 'observed' frequencies in each of the three components $\left(I_{j(-)}^{k}\right)$ $\left.I_{j(0)}^{k}, I_{j(+)}^{k}\right)$ obtained from $\boldsymbol{\gamma}^{k}$. If there is prior belief that a covariate $x_{j}$ has a negative/null/positive impact in most or all sub-populations, this could be incorporated by selecting a larger value for $a_{j(-)} / a_{j(0)} / a_{j(+)}$.

2. Subsequently, the latent variables are sampled for each covariate and sub-population from a discrete distribution with probabilities

$$
p\left(\gamma_{j(g)}^{k}=d \mid \mathbf{y}, \boldsymbol{\beta}^{k}, \sigma^{k}, \boldsymbol{\pi}_{j}^{k}\right)= \begin{cases}s_{(-)} /\left(s_{(-)}+s_{(0)}+s_{(+)}\right) & \text {if } d=-1 \\ s_{(0)} /\left(s_{(-)}+s_{(0)}+s_{(+)}\right) & \text {if } d=0 \\ s_{(+)} /\left(s_{(-)}+s_{(0)}+s_{(+)}\right) & \text {if } d=+1\end{cases}
$$

where the elements that determine the probabilities are

$$
\begin{aligned}
& \boldsymbol{s}_{(-)}=\pi_{j(-)}^{k} p\left(\mathbf{y}_{(g)} \mid \boldsymbol{\beta}_{-j(g)}^{k}, \beta_{j(g)}^{k}, \sigma^{k}\right) p\left(\beta_{j(g)}^{k} \mid \mu_{j(-)}^{k}, \sigma_{j(-)}^{2, k}\right) \\
& \boldsymbol{s}_{(0)}=\pi_{j(0)}^{k} p\left(\mathbf{y}_{(g)} \mid \boldsymbol{\beta}_{-j(g)}^{k}, \beta_{j(g)}^{k}=0, \sigma^{k}\right) \\
& \boldsymbol{s}_{(+)}=\pi_{j(+)}^{k} p\left(\mathbf{y}_{(g)} \mid \boldsymbol{\beta}_{-j(g)}^{k}, \beta_{j(g)}^{k}, \sigma^{k}\right) p\left(\beta_{j(g)}^{k} \mid \mu_{j(+)}^{k}, \sigma_{j(+)}^{2, k}\right) .
\end{aligned}
$$


The vector $\boldsymbol{\beta}_{-j(g)}^{k}$ contains all regression coefficients except $\beta_{j(g)}^{k}$ and $\mathbf{y}_{(g)}$ corresponds to the response of the individuals in sub-population $g$. Notice that $p(\mathbf{y} \mid \boldsymbol{\beta}, \sigma)=N\left(\mathbf{X} \boldsymbol{\beta}, \sigma^{2} I\right)$.

3. Next, the regression coefficients are obtained by taking $\beta_{j(g)}^{k}=0$ for all covariates in group $g$ where $\gamma_{j(g)}^{k}=0$ and sampling the other coefficients with $\gamma_{j(g)}^{k} \neq 0$ in sub-population $g$ as $\boldsymbol{\beta}_{-0(g)}^{k} \sim N\left(\boldsymbol{\mu}_{-0(g)}^{k}, \sigma^{2, k} \boldsymbol{\Delta}_{-0(g)}^{k}\right)$ where $\boldsymbol{\Delta}_{-0(g)}^{k}=$ $\left(\mathbf{X}_{-0(g)}^{\prime} \mathbf{X}_{-0(g)}+\boldsymbol{\Delta}_{-0(g)}\right)$ with $\mathbf{X}_{-0(g)}$ containing the covariates for which $\gamma_{j(g)}^{k} \neq 0$ and the matrix $\boldsymbol{\Delta}_{-0(g)}$ is diagonal with $1 / \sigma_{j(-)}^{2, k}$ when $\gamma_{j(g)}^{k}=-1$ and $1 / \sigma_{j(+)}^{2, k}$ if $\gamma_{j(g)}^{k}=+1$. The mean vector is $\boldsymbol{\mu}_{-0(g)}^{k}=\left(\boldsymbol{\Delta}_{-0(g)}^{k}\right)^{-1}\left(\mathbf{X}_{-0(g)}^{\prime} \mathbf{y}+\boldsymbol{\Delta}_{-0(g)} \boldsymbol{\mu}_{-0(g)}\right)$ where $\boldsymbol{\mu}_{-0(g)}$ is a vector with $\mu_{j(-)}^{k}$ when $\gamma_{j(g)}^{k}=-1$ and $\mu_{j(+)}^{k}$ if $\gamma_{j(g)}^{k}=+1$.

4. Sample the other parameters from $p\left(\boldsymbol{\mu}_{(-)}, \boldsymbol{\mu}_{(+)}, \sigma_{(-)}, \sigma_{(+)}, \mathbf{r}_{(-)}, \mathbf{r}_{(+)}, \sigma \mid \mathbf{y}, \boldsymbol{\beta}\right)$. These parameters were not assigned conditionally conjugate priors, so the conditional distributions do not present closed forms. Therefore, exploration of the posterior distribution for these parameters requires algorithms such as slice samplers or Metropolis-Hastings. We implement the BADE approach in JAGS (Plummer, 2013), which explores the parameters univariately via the slice sampler.

A Gibbs sequence is obtained by repeating the above sampling procedure. In the BADE approach, we classify the effect of each covariate across sub-populations based on the sampled $\boldsymbol{\gamma}^{1}, \ldots, \boldsymbol{\gamma}^{m}$. The posterior probability that the effect of $x_{j}$ in sub-group $g$ is negative can be computed as $\sum_{k} I\left\{\gamma_{j(g)}^{k}=-1\right\} / m$, whereas the posterior probability of $\beta_{j(g)}=0$ is $\sum_{k} I\left\{\gamma_{j(g)}^{k}=0\right\} / m$ and the posterior probability that this effect is positive can be computed as $\sum_{k} I\left\{\gamma_{j(g)}^{k}=+1\right\} / m$. The sum of these three probabilities is

$$
p\left(\beta_{j(g)}<0 \mid \mathbf{y}\right)+p\left(\beta_{i(g)}=0 \mid \mathbf{y}\right)+p\left(\beta_{j(g)}>0 \mid \mathbf{y}\right)=\sum_{d \in\{-1,0,+1\}} p\left(\gamma_{j(g)}^{k}=d \mid \mathbf{y}\right)=m / m=1,
$$

showing appropriateness of the posterior distribution. Similarly, given that the vector $\left(\pi_{j(-)}, \pi_{j(0)}, \pi_{j(+)}\right)$ is assigned a Dirichlet distribution, the sum of the prior distributions $p\left(\beta_{j(g)}<0\right)+p\left(\beta_{j(g)}=0\right)+p\left(\beta_{j(g)}>0\right)=\pi_{j(-)}+\pi_{j(0)}+\pi_{j(+)}=1$. Based on posterior inference, we establish the classification of the interactions which is most supported by the data.

We classify each interaction $\beta_{j(g)}$ individually based on $\gamma_{j(g)}^{1}, \ldots, \gamma_{j(g)}^{m}$. Following the median probability model approach of Barbieri et al. (2004), the effect of $x_{j}$ in sub-group $g$ is classified as negative if the posterior probability of $\gamma_{j(g)}=-1$ is at least $1 / 2$. Similarly, if the proportion of Gibbs iterations where $\gamma_{i(g)}=+1$ is greater than 
or equal to $1 / 2$, the effect is classified as positive. In any other case, we conclude that there is no impact of covariate $x_{j}$ in sub-group $g$.

\section{Simulation study}

In this section, we study the performance of the BADE approach via simulations. The first example illustrates the advantages of the method in a case with a large number of sub-populations, whereas the second example involves few sub-groups. All methods were implemented in JAGS (Plummer, 2013) and run from R (R Core Team, 2018) with the rjags package. The code is available as supplementary material.

We estimate all models sampling three chains and taking 10000 iterations as burn-in, followed by 20000 additional iterations thinned by a factor of 20. Convergence of the MCMC samples was assessed based on the Brooks-Gelman-Rubin (BGR) diagnostic (Lesaffre and Lawson, 2012). If the value of BGR was higher than 1.2 for some of the parameters in the model, an additional 20000 iterations were sampled until obtaining convergence.

The proposed BADE method is assessed based on the total number of group interactions $\beta_{j(g)}$ that are misclassified. A group interaction is misclassified if: (a) the interaction coefficient is generated as null in the simulation and the procedure indicates an important effect, (b) the interaction is generated as $\beta_{j(g)} \neq 0$ and the method suggests no effect of the regressor in that sub-group, and finally, (c) the covariate effect in the sub-group is non-zero and the method detects it but with the wrong sign. Additionally, we study the bias and mean squared error of the regression coefficients for the methods under comparison.

\subsection{A large number of sub-populations}

The performance of the BADE method is compared with that of fitting a mixed-effects model when the number of sub-groups is large $(G=100)$. The response was generated using the following model with 15 covariates

$$
y_{i(g)}=\beta_{1(g)} x_{1, i(g)}+\beta_{2(g)} x_{2, i(g)}+\cdots+\beta_{15(g)} x_{15, i(g)}+\varepsilon_{i(g)},
$$

with $n_{g}=20$ observations for each sub-population. To simulate differential effects, the interaction coefficients were generated from a mixture of two uniform distributions and a spike at zero. Namely,

$$
\beta_{i(g)} \sim 0.5 \times U(-3,-2)+0.2 \times I\left\{\beta_{j(g)}=0\right\}+0.3 \times U(2,3),
$$

for $j=1, \ldots, 15$ and $g=1, \ldots, 100$. Notice that this mixture is not symmetric, reflecting that in practice, for a given covariate, it is likely to have more sub-groups in one of the two components (positive or negative signals). We study the case where the coefficients come from a symmetric distribution in Section 4.2. 
The covariates are generated from a standard normal distribution, and the residuals follow $\varepsilon_{i} \sim \mathcal{N}\left(0, \sigma^{2}\right)$ with $\sigma=\{1,2,3,4,5\}$ to vary the signal-to-noise ratio. We generated 100 replicated datasets from model (4.1). Regarding the hyperparameters in BADE, we take $b=c=0.01$ for $\sigma^{2}$ and $r=\{1,4,9\}$ for $r_{j(-)}$ and $r_{j(+)}$ in (2.3). As shown in the appendix, the performance of BADE was similar for the three values of $r$, so the results are presented for $r=9$ in the simulation studies and the application in Section 5. For the probability vectors we take $\pi_{j} \sim \operatorname{Dir}\left(a_{j(-)}, a_{j(0)}, a_{j(+)}\right)$ with $a_{j(-)}=a_{j(0)}=a_{j(+)}=1$, which leads to a uniform prior since $p\left(\pi_{j}\right) \propto \pi_{j(-)}^{a_{j(-)}^{-1}} \pi_{j(0)}^{a_{j(0)}-1} \pi_{j(+)}^{a_{j(+)}-1}$.

In order to assess the BADE approach, we fit a random-effects model including one random-effect for each covariate, that is, $b_{j(g)} \sim N\left(\beta_{j}, \tau_{j}^{2}\right)$ for $j=1, \ldots, 15$ and $g=$ $1, \ldots, 100$. Similarly as in Gelman et al. (2012), the interactions are classified based on the $95 \%$ credible interval of the random-effects $b_{j(g)}$. Given that the interactions in (4.1) are generated independently, we assume independence among random-effects to avoid estimating the covariance matrix of dimension $15 \times 15$.

The mixed model is estimated taking a uniform density for the standard deviation of the random-effects as suggested by Gelman (2006), specifically $\tau_{j} \sim U(0,1)$. We selected this range for $\tau_{j}$ given that in the BADE approach, the covariates and the response are rescaled in units of standard deviation. For the fixed effects we select $\beta_{j} \mid \sigma \sim U\left(r_{j(-)} \sigma, r_{j(+)} \sigma\right)$ with $r_{j(-)} \sim N^{-}(0, r)$ and $r_{j(+)} \sim N^{+}(0, r)$, similarly as in BADE. Finally, to study if the interactions in (4.1) may be better described by a heavy-tailed distribution, we fit a mixed model assuming that the random-effects $b_{j(g)}$ follow a $t$-distribution. When estimating this heavy-tailed mixed model, we assign a uniform prior distribution $U(3,100)$ to the degrees of freedom of the $t$-distribution.

Figure 2 presents the average number of misclassified interactions (out of the $15 \times 100=1500$ ) for the three approaches. As we can see, there are no important differences between the mixed model assuming normality for the random-effects and the heavy-tailed mixed model. On the other hand, the BADE method is clearly superior to the multilevel models, which perform partial pooling for all sub-groups and shift the estimates towards each other.

In Figure 3, we present the average absolute bias and the mean squared error for the regression coefficients. Once more, the BADE approach outperforms the random-effects models. Our proposal combines the information only among sub-populations with a similar covariate effect, which improves the identification of important effects and the estimation of such effects. Estimation of the mixed model assuming normality for the random-effects requires about 7 minutes on a quad-core processor $3.0-\mathrm{GHz}$ laptop, whereas the heavy-tailed mixed model takes around 216 minutes and the BADE approach requires about 126 minutes. Let us recall that in this example we have a large number of observations and covariates. 


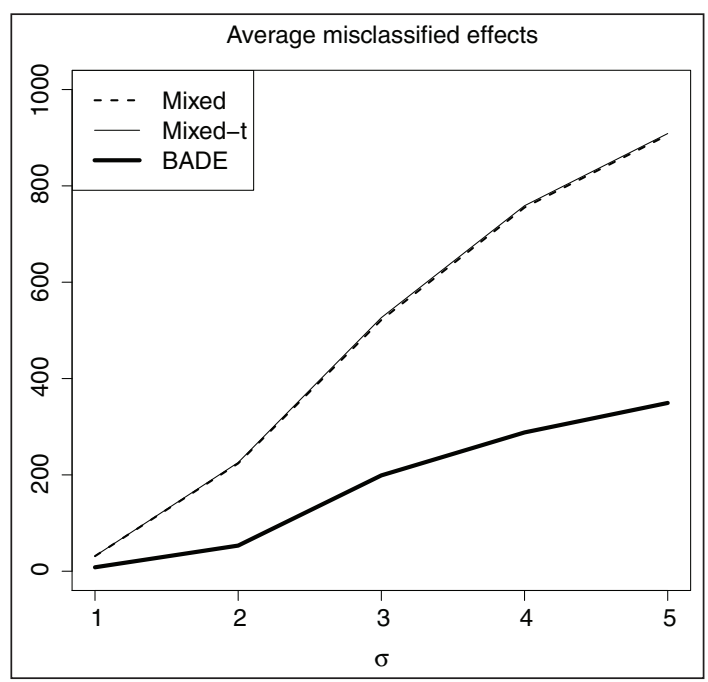

Figure 2 Average number of misclassified interactions in the 100 sub-groups. The results are presented for the mixed-effects models and the BADE approach

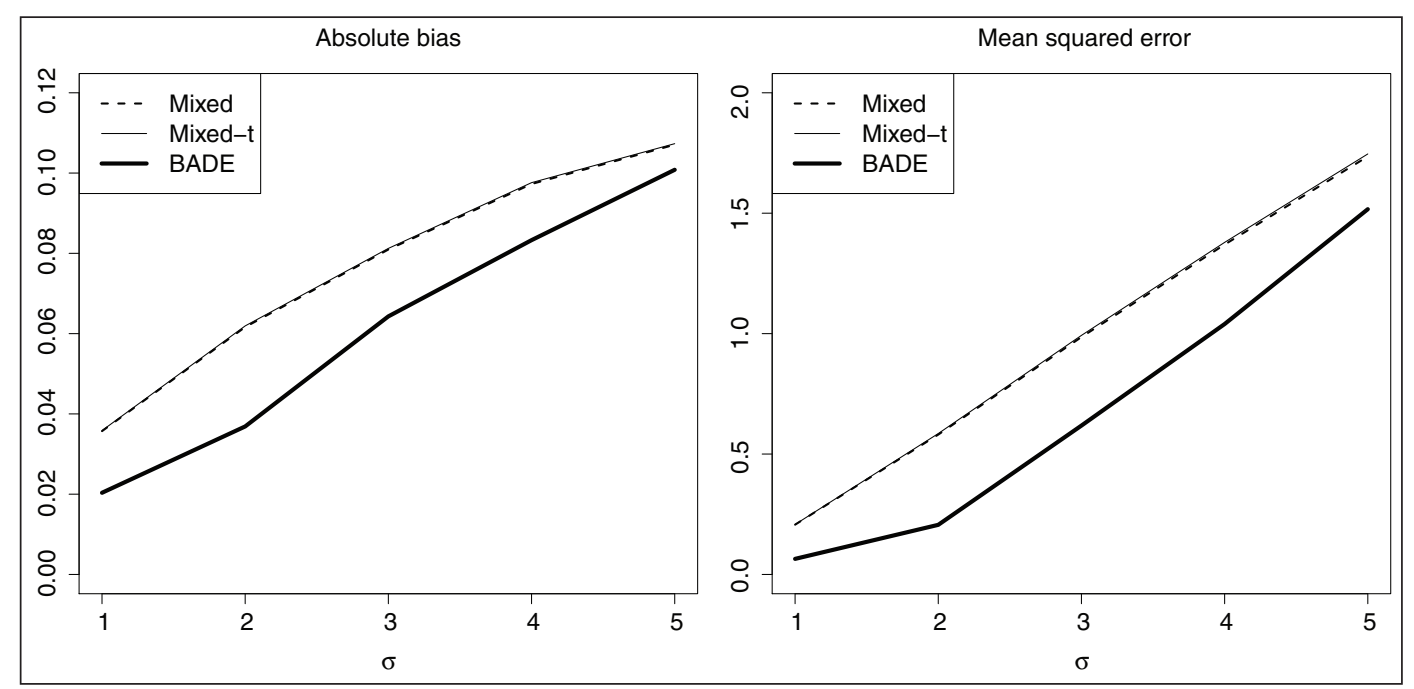

Figure 3 Average absolute bias and mean squared error for the interactions in the 100 sub-groups. The results are presented for the mixed-effects models and the BADE approach

\subsection{Performance for few sub-populations}

The BADE approach was designed for cases with many sub-populations, but it is of interest to study its performance in situations with few sub-groups. In this example, the data are generated assuming $G=6$ sub-groups and a model with three covariates. 


\section{Adrian Quintero et al.}

More specifically,

$$
y_{i(g)}=\beta_{0(g)}+\beta_{1(g)} x_{1, i(g)}+\beta_{2(g)} x_{2, i(g)}+\beta_{3(g)} x_{3, i(g)}+\varepsilon_{i(g)},
$$

with $n_{g}=50$ observations for each sub-population $g=1, \ldots, 6$. The regression coefficients are generated from a normal distribution, that is, $\beta_{j(g)} \sim \mathcal{N}\left(0,0.75^{2}\right)$. The coefficients with values smaller than one quarter of the standard deviation in absolute value $\left(\left|\beta_{j(g)}\right|<0.75 / 4\right)$ were assigned the value zero. This means that around $20 \%$ of the coefficients are null, which corresponds to the sub-populations where the covariates have no effect.

As before, the covariates are generated from a standard normal distribution and the residuals follow $\varepsilon_{i} \sim \mathcal{N}\left(0, \sigma^{2}\right)$ with $\sigma=\{1,2,3,4,5\}$. In order to assess the BADE approach, we fit a mixed model which combines the information across sub-groups, and the effects are classified similarly as in Section 4.1. Additionally, we classify the coefficients of the full interaction model (1.2) based on the three-component mixture prior advocated by Conlon (2008):

$$
p\left(\beta_{j(g)} \mid \gamma_{j(g)}\right)= \begin{cases}\mathcal{N}^{-}\left(0, r \sigma^{2}\right) & \text { if } \gamma_{j(g)}=-1 \\ 1 \times I\left\{\beta_{i(g)}=0\right\} & \text { if } \gamma_{j(g)}=0 \\ \mathcal{N}^{+}\left(0, r \sigma^{2}\right) & \text { if } \gamma_{j(g)}=+1\end{cases}
$$

where the latent variables follow $\gamma_{j(g)} \sim \operatorname{Multinomial}\left(1 ; \pi_{(-)}, \pi_{(0)}, \pi_{(+)}\right)$. Conlon (2008) showed via simulations that this three-component mixture outperforms the two-component spike-slab prior of Mitchell and Beauchamp (1988) in the context of microarray studies. However, notice that the three-component mixture of Conlon (2008) does not combine the information across sub-populations with a similar covariate effect.

We generated 100 replicated datasets from model (4.2) to compare the three approaches. Figure 4 presents the average number of misclassified interaction effects as a function of the variance of the error. As we can see, the Conlon model (4.3) is outperformed by the other two methods, which combine information across sub-populations. In addition, the BADE approach performs better than the mixed model even though the coefficients in model (4.2) were generated based on a normal density, which is the assumed distribution in random-effects models. This reflects the fact that mixed models are not designed to classify the random-effects, and the choice of the credible intervals' size is arbitrary.

Figure 5 presents the average absolute bias and the mean squared error for the regression coefficients. We can see that Conlon model (4.3) leads to low accuracy for the estimators, since it does not combine the information across sub-groups. On the other hand, the BADE approach presents similar accuracy as the mixed model. Regarding the computational time for estimation, the Conlon model (4.3) requires about 7 minutes, whereas the mixed model takes around 1 minute and the BADE approach requires about 3 minutes. 


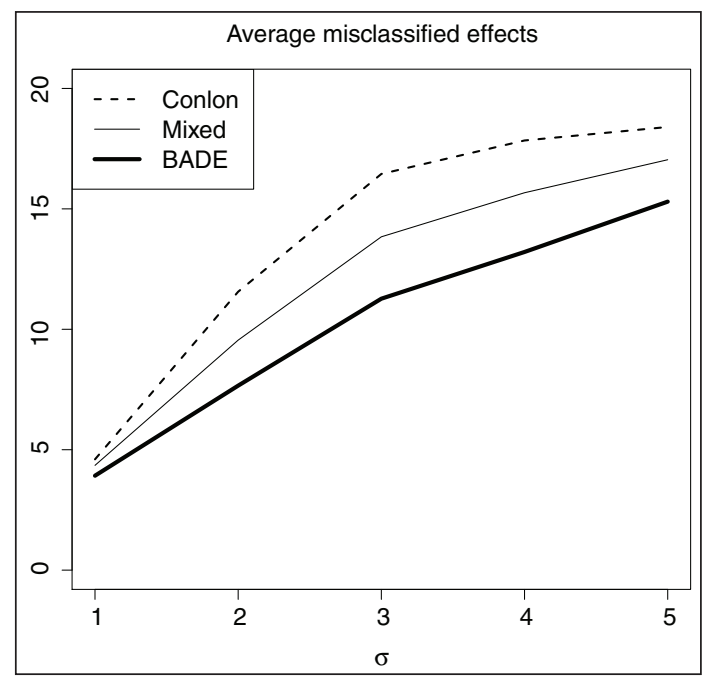

Figure 4 Average number of misclassified interactions in the six sub-groups. The results are presented for the Conlon model (4.3), the BADE approach and mixed model

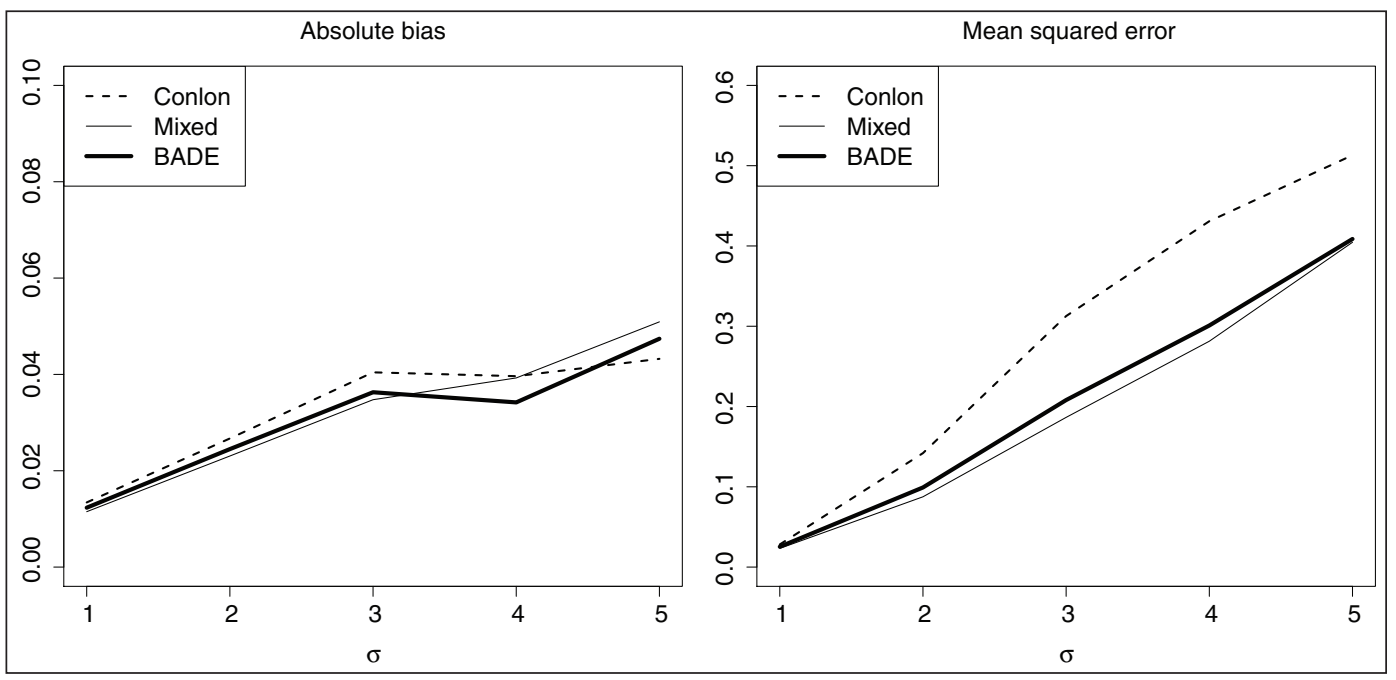

Figure 5 Average absolute bias and mean squared error for the interactions in the six sub-groups. The results are presented for the Conlon model (4.3), the BADE approach and mixed model

\section{Patient satisfaction with hospital care}

The registered nurse forecasting (RN4CAST) study was conducted between 2009 and 2011, see Sermeus et al. (2011) for details. Using the Hospital Consumer Assessment of Healthcare Providers and Systems (HCAHPS; Darby et al., 2005), patients from 
seven countries were asked before discharge to rate their hospital on a scale from 0 (worst) to 10 (best) as a measure of overall satisfaction. Additionally, they completed 16 survey items reflecting specific patient experiences related to nurse communication (Q1-Q3), doctor communication (Q5-Q7), responsiveness (Q4, Q10), physical environment $(\mathrm{Q} 8, \mathrm{Q} 9)$, pain control $(\mathrm{Q} 11, \mathrm{Q} 12)$, medicine communication $(\mathrm{Q} 13$, Q14) and discharge information (Q15, Q16). See the supplementary material for the detailed wording of the survey items. There are in total 3046 patients with complete information clustered in 186 hospitals.

The items that are influential on patient satisfaction may differ according to many factors. For instance, the geographical regions in each country present cultural differences that plausibly influence satisfaction standards of the inhabitants. In addition, depending on the speciality of the medical centre (paediatrics, mental health, surgery, etc.), there are diverse types of patients treated and the aspects considered as crucial for satisfaction may differ. Thus, it is of interest to determine for each hospital which patient experiences (reflected in the 16 items) have an impact on the overall rating. The results from such analysis are relevant to define hospital-specific policies that improve patient's satisfaction with care.

The full interaction model (1.2) is not identifiable in this case given that $67 \%$ of the hospitals have less than 16 patients. Hence, the BADE method was implemented with a Gibbs sequence of $m=100000$ iterations, after discarding 10000 iterations as burn-in. Table 1 presents the percentage of hospitals within each country where the association between the covariate (specific patient experiences) and the outcome (overall rating) is positive. Negative associations were found in extremely few cases $(0.4 \%$ of the instances).

The findings of the BADE method suggest that in many instances the association between specific patient experiences and the overall rating was positive in the majority of hospitals, with some specific experiences showing a positive association in all hospitals within a country and with almost none to very little variation across countries. This is the case for the first two items on nurse communication that relate to being treated with courtesy and respect and being listened to (Q1, Q2), being helped as soon as expected (Q4), doctors treating patients with courtesy and respect $(\mathrm{Q} 5)$, having a quiet room at night $(\mathrm{Q} 9)$ and being told what medicines were for (Q13). Other specific experiences showed more ambiguity across hospitals concerning positive associations with the overall rating (Q3, Q7, Q8 and Q15), while others showed a positive association in very few hospitals only (Q6, Q10, Q11, Q12, Q14 and Q16).

We present a more detailed analysis in the supplementary material with the profile of the hospital with the lowest average rating in each country. The sign of the coefficient is depicted for the covariates that are identified as important in each hospital for the 16 items of interest. Such analysis can help to improve satisfaction in low performing hospitals.

We fitted a mixed model and classified the effects similarly as in Section 4.1. Table 2 suggests that there are very few items with a positive impact across hospitals based on this approach. These results are not in agreement with previous research on patient satisfaction (Boulding et al., 2011). To analyse this in more detail, we 
Table 1 Percentage of hospitals within each country where the association between the items and overall satisfaction is positive, using the BADE approach

\begin{tabular}{lccccccc}
\hline & Belgium & Switz. & Spain & Finland & Greece & Ireland & Poland \\
\hline Q1 & 93 & 95 & 87 & 88 & 100 & 100 & 87 \\
Q2 & 100 & 100 & 93 & 97 & 100 & 100 & 100 \\
Q3 & 42 & 45 & 47 & 31 & 35 & 60 & 57 \\
Q4 & 95 & 100 & 93 & 100 & 100 & 90 & 93 \\
Q5 & 73 & 86 & 53 & 62 & 88 & 90 & 67 \\
Q6 & 45 & 32 & 7 & 31 & 65 & 60 & 43 \\
Q7 & 32 & 36 & 20 & 19 & 29 & 40 & 63 \\
Q8 & 27 & 27 & 20 & 28 & 41 & 70 & 43 \\
Q9 & 98 & 100 & 93 & 97 & 100 & 100 & 100 \\
O10 & 2 & 5 & 40 & 3 & 6 & 10 & 33 \\
Q11 & 2 & 5 & 7 & 0 & 0 & 0 & 0 \\
Q12 & 7 & 18 & 7 & 12 & 6 & 40 & 17 \\
O13 & 88 & 95 & 93 & 91 & 100 & 90 & 90 \\
Q14 & 12 & 23 & 27 & 6 & 6 & 10 & 27 \\
Q15 & 18 & 18 & 7 & 25 & 12 & 30 & 53 \\
O16 & 25 & 36 & 13 & 25 & 0 & 10 & 50 \\
\hline
\end{tabular}

Table 2 Percentage of hospitals within each country where the association between the items and overall satisfaction is positive, using the mixed model

\begin{tabular}{lccccccc}
\hline & Belgium & Switz. & Spain & Finland & Greece & Ireland & Poland \\
\hline Q1 & 0 & 5 & 0 & 6 & 0 & 0 & 23 \\
Q2 & 40 & 36 & 47 & 34 & 35 & 70 & 70 \\
Q3 & 0 & 0 & 0 & 0 & 0 & 0 & 10 \\
Q4 & 2 & 5 & 7 & 3 & 0 & 0 & 23 \\
Q5 & 0 & 0 & 7 & 0 & 0 & 10 & 20 \\
Q6 & 0 & 0 & 0 & 0 & 0 & 0 & 10 \\
Q7 & 0 & 0 & 0 & 0 & 0 & 0 & 0 \\
Q8 & 5 & 0 & 0 & 0 & 0 & 0 & 17 \\
Q9 & 5 & 0 & 7 & 0 & 0 & 0 & 13 \\
Q10 & 0 & 0 & 7 & 3 & 0 & 0 & 20 \\
Q11 & 0 & 0 & 0 & 0 & 0 & 0 & 0 \\
Q12 & 2 & 0 & 0 & 0 & 0 & 0 & 7 \\
Q13 & 0 & 0 & 0 & 0 & 0 & 0 & 3 \\
O14 & 0 & 0 & 0 & 0 & 0 & 0 & 3 \\
Q15 & 0 & 0 & 0 & 3 & 0 & 0 & 3 \\
Q16 & 0 & 0 & 0 & 0 & 0 & 0 & 0 \\
\hline
\end{tabular}

compare the estimated regression coefficients for all items based on BADE and the mixed model in Figure 6. As we can see, several coefficients are estimated to be around 0.5 or higher based on BADE, whereas the mixed model estimates them to be smaller than 0.4 . The latter method performs partial pooling for all hospitals and shrinks most of the largest coefficients, whereas our BADE approach combines the information only for hospitals with similar covariate effects.

Nevertheless, the differences between the estimates from both methods in Figure 6 do not explain why the mixed model classifies the effect of most items as null across 


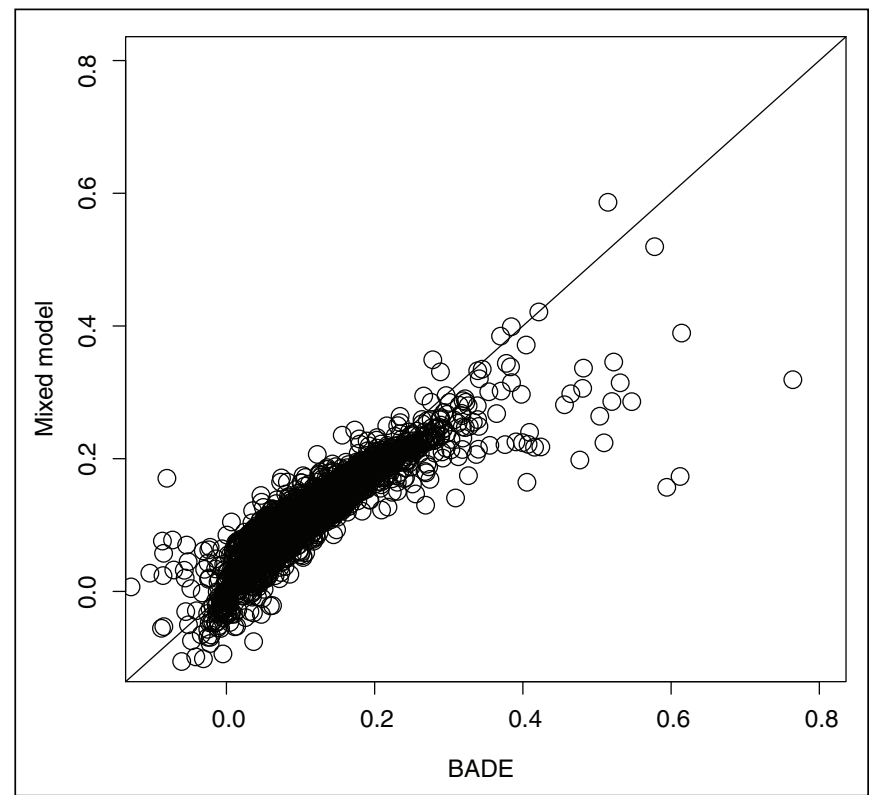

Figure 6 Comparison of estimated regression coefficients based on BADE and a mixed model. The estimates are presented for all covariates and all hospitals in the study

countries. For instance, when looking at the Belgian hospital with the lowest average satisfaction score (in supplementary material), the estimates for the first two items present the same value for both methods, 0.11 for Q1 and 0.18 for Q2. However, the $95 \%$ credible intervals according to the mixed model are $(-0.10,0.22)$ and $(-0.04,0.22)$, leading to consider those effects as non-important, while BADE and the literature on patient satisfaction indicate that such items are important. Indeed, the mixed model is not designed to classify the random-effects. The size of the credible intervals is chosen arbitrarily, taking a $95 \%$ probability level which is commonly used in frequentist statistics. On the other hand, our BADE approach is designed to classify the regression coefficients while combining the information across sub-populations. Moreover, the simulations showed that BADE performs significantly better than the mixed model.

\section{Discussion}

We have introduced a Bayesian methodology to identify important covariate effects in studies with multiple sub-populations. The approach was motivated in the case of many sub-groups such as hospitals or other clustering units, but the simulations show that combining the information across sub-populations is advantageous in situations with few sub-groups as well. Therefore, the BADE method can be applied in numerous fields that consider the interaction model (1.2). 
For instance, a concern in Bayesian sub-group analysis for clinical trials (Pocock et al., 2002; Liu et al., 2017) is that dividing the whole sample of patients and considering model (1.2) leads to larger variability in the estimation of the regression coefficients, making it more difficult to detect important effects. The proposed BADE method can help identify important interactions more efficiently since the information is combined across sub-groups of patients with similar covariate effects.

The BADE approach was presented without selecting a baseline sub-group in the full interaction model (1.2). However, if there is specific interest in comparing the sub-populations with a pre-selected reference group, the method can be adapted by modifying (2.1) such that for each covariate, when $\gamma_{j(g)}=0$, the interactions are equal to the effect in the reference group $\left(\beta_{i(g)}=\beta_{i(r)}\right)$, and when $\gamma_{i(g)}=-1(+1)$, the coefficient is $\beta_{j(g)}<\beta_{j(r)}\left(\beta_{j(g)}>\beta_{j(r)}\right)$. In this way, the results of the BADE method indicate in which sub-populations the effect of each covariate is lower, similar or higher compared to the reference group.

The simulation study showed that BADE can perform better than mixed models in identifying covariate effects across sub-populations. In the analysis of patient satisfaction, the estimates from the mixed model were similar to those from BADE in many instances. However, with the mixed model the credible intervals for the random-effects were rather wide, leading to most covariate effects being classified as null across hospitals. Indeed, mixed models are not designed to classify the random-effects and the choice of the credible intervals' size is arbitrary.

In order to analyse the impact of heteroscedasticy in the data, we repeated the simulation study in Section 4.2 but assuming different residual variance across sub-populations. For this, we generated the replicated datasets from model (4.2) assuming that $\varepsilon_{i(g)} \sim \mathcal{N}\left(0, \sigma_{g}^{2}\right)$ with $\sigma_{g} \sim U(1,5)$. For the three models studied in Section 4.2, we found that the absolute bias and the mean squared error increased between $10 \%$ and $20 \%$ compared to the homoscedastic case with $\sigma=3$, which is the mean of $\sigma_{g}$. Therefore, a possible extension of the method could involve heteroscedasticity, but this requires to modify the prior specification of BADE, especially for $\mu_{j(-)}$ and $\mu_{j(+)}$ in (2.2).

\section{Appendix}

\section{Prior specification for the mean components}

When taking as priors $\mu_{j(-)} \mid \sigma \sim N^{-}\left(0, r \sigma^{2}\right)$ and $\mu_{j(+)} \mid \sigma \sim N^{+}\left(0, r \sigma^{2}\right)$, a natural alternative to express lack of prior information for the means $\mu_{j(-)}$ and $\mu_{j(+)}$ would be to select a large value for $r$. However, the null model is favoured as $r \rightarrow \infty$, which is a form of the Bartlett-Lindley paradox (Bartlett, 1957).

This is reflected in the Gibbs procedure when there are covariates for which none of the sub-populations are classified in the negative (positive) component. As a consequence, the likelihood does not contribute with information to $\mu_{j(-)}\left(\mu_{j(+)}\right)$ 


\section{Adrian Quintero et al.}

in the MCMC procedure and the parameter is practically sampled from the prior. In that case, if $r \rightarrow \infty$, the sampled $\mu_{j(-)}\left(\mu_{j(+)}\right)$ can take an extreme value because the posterior conditional variance of the parameter equals $r \sigma^{2}$. Hence, it is unlikely that a sub-population gets classified in that component again in the following Gibbs iterations, since the corresponding probability in (3.2) will be close to zero for extreme values of $\mu_{j(-)}\left(\mu_{j(+)}\right)$. This leads to more sub-populations being classified in the null component $\gamma_{j(g)}=0$ for the interactions.

\section{Comparison of different values for $r$}

In Figure A.1, we present the number of misclasified interactions for the simulation study in Section 4.1 using different values for the hyperparameter $r$ in (2.3). The results are similar for $r \in\{1,4,9\}$ but slightly better for $r=9$. The absolute bias and mean squared error are exactly the same for the three values of $r$, so we do not present those plots because the lines superimpose each other.

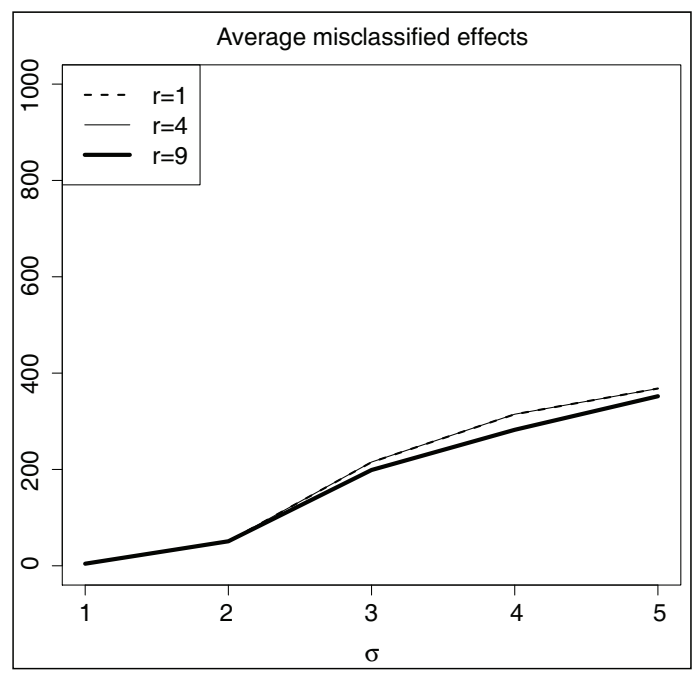

Figure A.1 Average number of misclassified interactions in the 100 sub-groups. The results are presented for different values of the hyperparameter $r$ in (2.3)

\section{Acknowledgements}

We are grateful to Jeroen Sichien and Walter Sermeus for their input in this article. We also thank the editor, referees and associate editor for their constructive comments and their input to improve this manuscript. 


\section{Declaration of conflicting interests}

The authors declared no potential conflicts of interest with respect to the research, authorship and/or publication of this article.

\section{Funding}

The authors received no financial support for the research, authorship and/or publication of this article.

\section{Supplementary material}

Supplementary materials for this article such as the code to fit the models in the simulation study, the dataset and the models in the application to patient satisfaction are available from http://www.statmod.org/smij/archive.html.

\section{References}

Barbieri MM and Berger JO (2003) Optimal predictive model selection. The Annals of Statistics, 32, 870-97.

Bartlett M (1957) A comment on D. V. Lindleys Statistical Paradox. Biometrika, 44, 533-34.

Boulding W, Glickman SW, Manary MP, Schulman KA and Staelin R (2011) Relationship between patient satisfaction with inpatient care and hospital readmission within 30 days. The American Journal of Managed Care, 17, 41-8.

Carlin B and Louis T (1996) Bayes and Empirical Bayes Methods for Data Analysis, 2nd edition. London: Chapman and Hall.

Conlon EM (2008) A Bayesian mixture model for metaanalysis of microarray studies. Functional and Integrative Genomics, 8, 43-53.

Darby C, Hays RD and Kletke P (2005) Development and evaluation of the CAHPS hospital survey. Health Services Research, 40, 1973-76.

Dixon DO and Simon R (1991) Bayesian subset analysis. Biometrics, 47, 871-81.
Gelman A (2006) Prior distributions for variance parameters in hierarchical models. Bayesian Analysis, 1, 515-34.

Gelman A, Hill J and Yajima M (2012) Why we (usually) don't have to worry about multiple comparisons. Journal of Research on Educational Effectiveness, 5, 189-211.

Ishwaran H and Rao JS (2005) Spike and slab gene selection for multigroup microarray data. Journal of the American Statistical Association, 100, 764-80.

Lesaffre E and Lawson A (2012) Bayesian Biostatistics (Statistics in Practice). Wiley: Chichester.

Liu J, Sivaganesan S, Laud PW and Muller P (2017) A Bayesian subgroup analysis using collections of ANOVA models. Biometrical Journal, 59, 746-66.

Mitchell TJ and Beauchamp JJ (1988) Bayesian variable selection in linear regression. Journal of the American Statistical Association, 83, 1023-32.

Pakes A (1983) Random group effects and the precision of regression estimates. Review of Economics and Statistics, 65, 168-73. 


\section{Adrian Quintero et al.}

Parmigiani G, Garrett ES, Anbazhagan R and Gabrielson E (2002) A statistical framework for expression-based molecular classication in cancer. Journal of the Royal Statistical Society: Series B (Statistical Methodology), 64, 717-36.

Plummer, M. (2013). Plummer M (2013) JAGS User Manual, 3.4.0 edition.

Pocock SJ, Assmann SE, Enos LE and Kasten LE (2002) Subgroup analysis, covariate adjustment and baseline comparisons in clinical trial reporting: current practice and problems. Statistics in Medicine, 21, 2917-30.

R Core Team (2018) R: A Language and Environment for Statistical Computing. R Foundation for Statistical Computing, Vienna, Austria. URL https://www.Rproject.org/

Sargent DJ and Hodges JS (1997) Smoothed anova with application to subgroup analysis. Research report, Department of Biostatistics, University of Minnesota, Minneapolis, $\mathrm{MN}$.

Schnell PM, Tang Q, Offen WW and Carlin BP (2016) A Bayesian credible subgroups approach to identifying patient subgroups with positive treatment effects. Biometrics, 72, 1026-36.

Sermeus W, Aiken L, Van den Heede K, Rafferty A, Griths P, Moreno-Casbas M, Busse R, Lindqvist R, Scott A, Bruyneel L, Brzostek T, Kinnunen J, Schubert M, Schoonhoven L, Zikos D and RN4CAST consortium (2011) Nurse forecasting in Europe (RN4CAST): rationale, design and methodology. $B M C$ Nursing, 10, 1-19.

Tanner M and Wong W (1987) The calculation of posterior distributions by data augmentation (with discussion). Journal of the American Statistical Association, 82, 528-40.

Verbeke G and Lesaffre E (1996) A linear mixed-effects model with heterogeneity in the random-effects population. Journal of the American Statistical Association, 91, 217-21.

Verbeke G and Molenberghs G (2000) Linear Mixed Models for Longitudinal Data, 1st edition. New York: Springer.

Zumbo B (2007) Three generations of DIF analyses: Considering where it has been, where it is now, and where it is going. Language Assessment Quarterly, 4, 223-33. 